\title{
An Insight into the Symptomatology of $\beta$-Thalassaemia Major: Molecular Genetic Basis of the Disease - III
}

\author{
Tahir Shamsi*, Saqib Ansari, Zeeshan Hussain \\ National Institute of Blood Disease \& Bone Marrow Transplantation, Karachi, Pakistan.
}

Thalassaemia syndrome is a group of inherited disorders resulting in low oxygen carrying capacity; severe anaemia due to haemolysis, ineffective erythropoiesis and bone marrow expansion in the bones, liver and spleen being the causes. Globally, there are approx. $394 \beta$ Thalassaemia mutations (excluding $\alpha$ and other variants genetic mutations) responsible for this disorder [1]. Each ethnic group has 5-6 common mutations causing $\beta$-thalassaemia; these mutations may not be shared in other ethnic groups [2]. Some mutations result in $\beta+$ phenotype (producing some functional $\mathrm{Hb}-\mathrm{A}$ while others have more severe $\beta^{0}$ phenotype (no functional $\mathrm{Hb}-\mathrm{A}$ is formed). Rare cases are caused by deletion in $\beta$ globin gene (deletion of 619 base pairs in exon-1 of $\beta$-globin gene); found in Gujrati, Sindhi, and Memon ethnicities.

Clinically, 40-50\% $\beta$-thalassaemia major patients (reported by different laboratories on the basis of Hb-F) are in fact have lesser clinical severity and present late. Despite of $\mathrm{Hb}-\mathrm{F}$ level above $90 \%$, have total haemoglobin level of more than $6.0 \mathrm{~g} / \mathrm{dl}$ at the time of haemoglobin electrophoresis and they present to the medical practitioners after two year of age [2-4]. This has serious implications on their life long management. Correct diagnosis at this point will help in avoiding regular blood transfusion and selection of patients who may benefit from haemoglobin augmenting agents and good supportive care. There are multiple genetic factors responsible for lesser degree of severity in Pakistani $\beta$-thalassaemia population. We will discuss some of these factors in this series of editorials on the "Insight into the Symptomatology of $\beta$-Thalassaemia Major".

Mutations (genotypes-phenotypes): The most common $\beta$-globin gene mutations responsible for spread of $\beta$-thalassaemia major convering upto 93\% of all patients in Pakistan are IVS 1-5, Frameshift 8-9, Deletion of 619 base pairs in exon-1, IVS 1-1, Frameshift 41-42, and Codon 30. Others like Cap+1 mutation in the promoter region, Codon 5, Codon-15 and others are uncommon or rare. Of all these, IVS1-5 is the most common mutation in all-ethnic groups. In many patients, it is responsible for $\beta+$ phenotype; these patients present late, have a higher total haemolgobin and have moderate to massive hepato-splenomegaly on presentation. In Balochi cohort, it is present in up to $80 \%$ patients. Second most common mutation is Frameshift 8-9. It is commonly seen Punjabi, Seraiki and Pushto speaking population. It is a $\beta^{0}$ mutation and has a very severe phenotype. Deletional type $\beta$-thalassaemia (deletion of 619 base pairs in exon-1 of $\beta$-gobin gene) is found in Gujrati, Sindhi, and Memon ethnicities. It is less common or rare in other ethnic groups. It is a severe phenotype and has a $\beta^{0}$ variety. Other less common mutations like IVS 1-1, Frameshift 41-42, and Codon 30 have $\beta^{0}$ severe phenotype. Cap +1 mutation is a slient mutation and present as a milder phenotype, does not cause higher $\mathrm{Hb}-\mathrm{A} 2$ in carriers (they are reported as non-carriers). Usually, patients carrying this mutation require minimal blood transfusion.

Co-inheritance of $\alpha$-thalassaemia is reported to be $30 \%$ in Pakistani $\beta$-thalassaemia patients [4, 5]. $80 \%$ are single $\alpha$-globin gene deletion while $19 \%$ are two gene deletion types. Remaining have triplicated $\alpha$-globin gene $(-\alpha \alpha \alpha)$. Over $95 \%$ alpha globin gene deletions are of 3.7 kilo base size while remaining is of 4.2 kilo bases. Other deletions are extremely rare. This coinheritance reduces the $\alpha$ :non- $\alpha$ globin chain imbalance and ameliorates the severity of $\beta$-thalassaemia; many are non-transfusion dependent $\beta$-thalassaemia. In fact $12 \%$ of Pakistani general population is reported to have $\alpha$-thalassaemia trait. This prevalence is higher than $\beta$-thalassaemia carrier state in this country.

Genetic modifiers like XMN-1 polymorphism (-158 C-T nucleotide change) in $\gamma$-globin gene [4] on chromosome 11, BCL11-A on short arm of chromosome 2 p16.1 and others have important bearings on producing $\gamma$-globin chains. In $\beta$-thalassaemia major, it provides a partner $\gamma$-globin chain to free $\alpha$-globin chain within the erythrocytes thereby making a normal / functional $\mathrm{Hb}-\mathrm{F}$. This is an important reason for delayed presentation of these patients because of maintaining a haemoglobin level of $6.0 \mathrm{~g} / \mathrm{dl}$ or more. In Pakistan, a total of $44 \%$ of $\beta$-thalassaemia major patients have XMN-1 polymorphism (in homozygous or heterozygous state). Its presence is a strong predictor for a response to haemoglobin $\mathrm{F}$ augmentation. On the other hand, BCL11A is down-regulated during hematopoietic cell differentiation. It has been found to play a role in suppressing Hb-F production. Therapeutic strategies aimed at increasing fetal globin production in diseases such as $\beta$ thalassemia by inhibiting BCL11A are currently being explored. In patients who have up-regulated BCL11 A, Hb-F production is suppressed and usually not a candidate of $\mathrm{Hb}-\mathrm{F}$ augmentation strategy. 
Knowledge of molecular genetic basis of an index patient at diagnosis greatly helps in planning the strategy to manage him clinically and counsel the family for prenatal diagnosis in future pregnancies. Unnecessary blood transfusion in up to $40 \%$ patients can be avoided $[6,7]$. Based on this information, patients should be selected for haemoglobin $\mathrm{F}$ augmentation therapy with hydroxyurea so that most of these patients may be benefited by this approach. Blindly using hydroxyurea where majority of patients will be non-responder disappoint patients, their families and the doctor prescribing it. Published data from Iran, India, and Pakistan on the use of hydroxyurea is very promising because of the genotype of $\beta$-thalassaemia in this region favours its benefits. On the contrary, genotype of $\beta$ thalassaemia from Fareast, Southeast Asia, China, and Mediteranean region have an entirely different genotype and response to hydroxyurea from these regions reported to be equivocal or disappointing.

We recommend utilizing the strength of molecular genetic testing at diagnosis. Whatever expenses are there, it will be once. But it will save millions by using appropriate treatment, minimal or no blood transfusion, greatly reducing the risk of transfusion transmitted infection, avoid iron overload and its life-threatening complications, and minimum requirement of iron chelation therapy.

\section{REFERENCES}

[1] ITHANET Portal. IthaGenes. [Online]. Available from: http://www.ithanet.eu/db/ithagenes?action=glist [Accessed 02 April 2018].

[2] Shabbir S, Nadeem M, Sattar A, et al. Type and frequency of haemoglobinopathies, diagnosed in the area of Karachi, in Pakistan. Cogent Med 2016; 3. DOI: 10.1080/2331205X.2016.1188875

[3] Ansari SH, Shamsi TS, Ashraf M, et al. Molecular epidemiology of $\beta$-thalassemia in Pakistan: Far reaching implications. Indian J Hum Genet 2012; 18(2): 193-7. DOI: 10.4103/0971-6866.100762

[4] Ansari SH, Shamsi TS, Munzir S, et al. G $\gamma$-Xmn I polymorphism: A significant determinant of $\beta$-thalassemia treatment without blood transfusion. J Pediatr Hematol Oncol 2013; 35(4): e153-6. DOI: 10.1097/MPH.0b013e31827e8662

[5] Shamsi T, Ansari S. Medical management of $\beta$-thalassaemia without blood transfusion: A myth or a reality? J Pak Med Assoc 2013; 63(3): 304-5.

[6] Shahid S, Nadeem M, Zahid D, Hassan J, Ansari S, Shamsi T. $\alpha$ thalassemia deletions found in suspected cases of $\beta$-thalassemia major in Pakistani population. Pak J Med Sci 2017; 33(2): 411-6. DOI: 10.12669/pjms.332.11834

[7] Ansari SH, Shamsi TS, Ashraf M, et al. Efficacy of hydroxyurea in providing transfusion independence in $\beta$-thalassemia. J Pediatr Hematol Oncol 2011; 33(5):339-43. DOI: 10.1097/MPH.0b013e31821b0770

\section{Editor-in-Chief}

Tahir Shamsi

National Institute of Blood Disease \& Bone Marrow Transplantation, Karachi, Pakistan 